\section{Colobronchial fistula following a partial resection of the colon}

MARINA MARAS-ŠIMUNIĆ • DRAGAN DRAGIČEVIĆ • MAJA ŠKORIĆ • NIKOLA KOLJA POLJAK • MARIN ŠIMUNIĆ
MARINA MARAS-ŠIMUNIĆ $(\bowtie)$ DRAGAN DRAGIČEVIĆ

Department of Diagnostic and Interventional Radiology University Hospital Split Šoltanska 1, 21000 Split, Croatia Phone: $+385 / 98 / 328-328$

Fax: +385/21/556-002

E-mail:mmaras@kbsplit.hr

MAJA ŠKORIĆ

Department of Radiology, General Hospital Knin, Knin, Croatia

NIKOLA KOLJA POLJAK Department of Otorhinolaryngology Head and Neck Surgery, University Hospital Center, Split, Croatia

MARIN ŠIMUNIĆ

School of Medicine, Split, Croatia

\begin{abstract}
We report a case of colobronchial fistula as a late consequence of the resection of the colon due to relapse of the gastrointestinal stromal tumor (GIST). A 54-year-old man experiencing pain in the left upper abdominal region underwent doublecontrast barium enema which revealed a fistulous channel between the splenic flexure of the colon and the bronchial tree. Fiberoptic bronchoscopy, after an extensive washout and aspiration of barium sulphate, confirmed the existence of a fistula in left lower subsegmental bronchi. The patient underwent left lower lobectomy, resection of the colobronchial fistula and resection of the splenic flexure of the colon. A year after the operation, the multidetector computed tomography (MDCT) showed neither signs of malignant abdominal disease, nor signs of pathological changes in the lung bases.
\end{abstract}

Key words: colobronchial fistula, barium enema, colon resection, postoperative complication.

\section{Introduction}

A fistula located between the colon and the bronchial tree is very rare. To the best of our knowledge, since the 1960s there have been only several reports in the literature that refer to that condition, most frequently associated with segmental colitis in Chron's disease. (1) Some cases of colobronchial fistulas are also reported as a consequence of trauma, (2) infections, (3) and malignant neoplasm. (4) Postoperative colobronchial fistula is exceptional, described in the literature as a complication of the coloesophageal interposition (5) and of splenectomy, partial gastrectomy and distal pancreatectomy. (6)

Colobronchial fistula is usually present as recurrent pneumonia, and is very difficult to diagnose, almost never after the onset of respiratory symptoms. In this paper we describe a case of colobronchial fistula as a late consequence of the surgery of malignant neoplasm of the colon.

\section{Case presentation}

A 54-year-old man was referred to our Department of Diagnostic and Interventional Radiology in order to clarify inexplicable pain in the left upper abdominal region lasting for several months. Eleven years prior to that moment he underwent gastrectomy, splenectomy and distal pancreatectomy due to the gastrointestinal stromal tumor (GIST) of the stomach, and due to the relapse of the GIST three years after that he also underwent the partial resection of the splenic flexure of the colon. Since then, the patient has been in continuous imatinib (Gleevec ${ }^{\circledR}$ ) therapy.

On the previous multidetector computed tomography (MDCT) (16row Somatom Sensation, Siemens; Erlangen, Germany) of the abdomen two months before, an infiltration in the posterobasal part of the left lower pulmonary lobe was identified, without signs of pathological changes in the abdomen (figure 1A). The patient 
was treated with broad-spectrum antibiotics for 20 days.

Double-contrast barium enema was performed (Polydoros 80s, Siemens; Erlangen, Germany) after regular preparation. First the barium sulphate was administered through the rectal tube until it filled the large intestine up to cecum. Afterwards, the patient expelled the surplus of the barium and the air was injected through the tube to expand the bowel lumen. Fluoroscopically and on abdominal plain films intestinal loops were appropriate in size, with smooth contours and without filling defects. Only after distension of the large bowel with air, a little trace of contrast showed up both spreading from the splenic flexure toward the lung bases (figure 1B) and filling the left-sided bronchial tree, trachea and even a part of the right-sided bronchi (figure 1C).

The examination was immediately terminated and the patient was taken to the Clinical Department of Pulmonary Diseases where urgent bronchoscopy was performed, under the admission diagnosis of suspected colobronchial fistula.

The examination was done with a flexible fiberoptic bronchoscope with real-time video equipment (Olympus Video Bronchoscope BF-P160; Olympus, Tokyo, Japan), following local anesthesia, while the patient was in a supine position. Vocal cords and upper trachea were regular. Barium was seen in the distal part of the trachea. A small amount of barium was seen in all bronchial branches on the right, however with abundant amounts in all branches on the left (figure 2). After an extensive washout and aspiration, a flush of foamy barium was noticed in the subsegmental bronchi of the posterior bronchus of the left lower lung lobe (left bronchus (LB) 10-branch b, and LB 9), indicating the location and confirming the existence of a colobronchial fistula. Edematous mucosa of these bronchi showed up after aspiration.

Except for a slight cough, our patient was in a good general condition, hemodynamically and respiratory stable. He was moved to the Clinic for thoracic surgery. Following preparation, a left thoracophrenotomy, left lower lobectomy, a resection of the colobronchial fistula and sutures of the large bowel at the splenic flexure were performed. The patient handled the operation well, and was given aimed antibiotic treatment along with intensive care in the postoperative period. Seven days after the surgery a purulent collection appeared in the thoracotomy area, indicating the revision of the wound. Clinically and radiologically, with some water soluble contrast, relapse was diagnosed as a colopleural fistulous communication; the patient underwent urgent reoperation. In the second operation a re-thoracophrenotomy, lung decortication and re-resection of the splenic flexure with laterolateral anastomosis were performed. His postoperative recovery, with intensive nutritional support, passed without complications this time, and the patient was discharged in a satisfactory condition.

Histopathological findings of surgical specimens revealed chronic inflammatory changes and multiple small abscesses in pulmonary parenchyma accreted with the fibrotic part of the diaphragm. The fistula channel between the colon and the bronchial tree was covered with squamous epithelium. Malignant cells have not been found either in lung parenchyma or in the fistula. During the pathological examination of the resected bowel, no malignant disease was identified

The follow-up abdominal MDCT six and twelve months later showed no signs of malignant disease or of pathological changes in the lung bases. Today, a year after operation of the fistula and twelve years after the first operation of the GIST of the stomach, our patient is in good general health.

\section{Discussion}

In the case of our patient, the fistula channel has been formed as a consequence of a partial resection

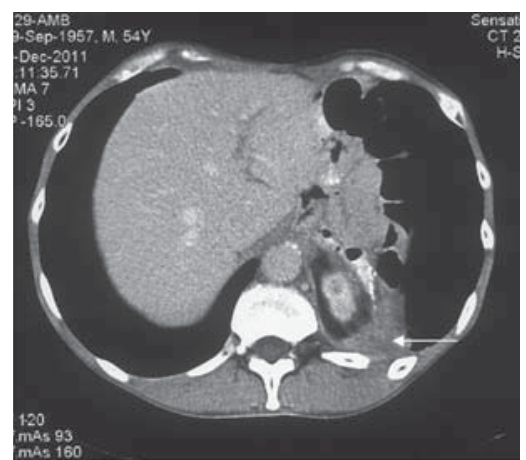

Figure 1A. Contrast enhanced abdominal multidetector computed tomography d e monstrating infiltration in the left lower pulmonary lobe and splenic flexure of the colon.

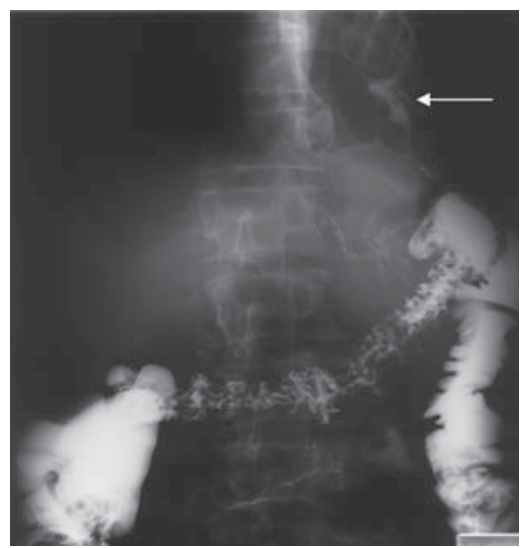

Figure 1 B. Abdominal plain film of double contrast barium enema showing fistula channel filled with barium arising from splenic flexure of the colon spreading towards the left lower lung base.

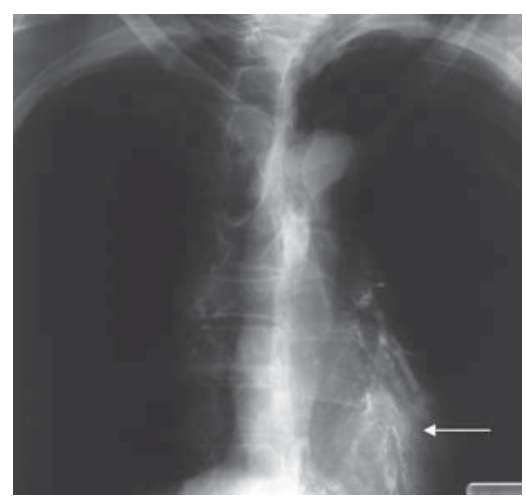

Figure $1 \mathrm{C}$. Chest $\mathrm{x}$-ray in supine position revealed barium in the left bronchial tree, and to a lesser extent in the trachea and the right bronchial tree. 

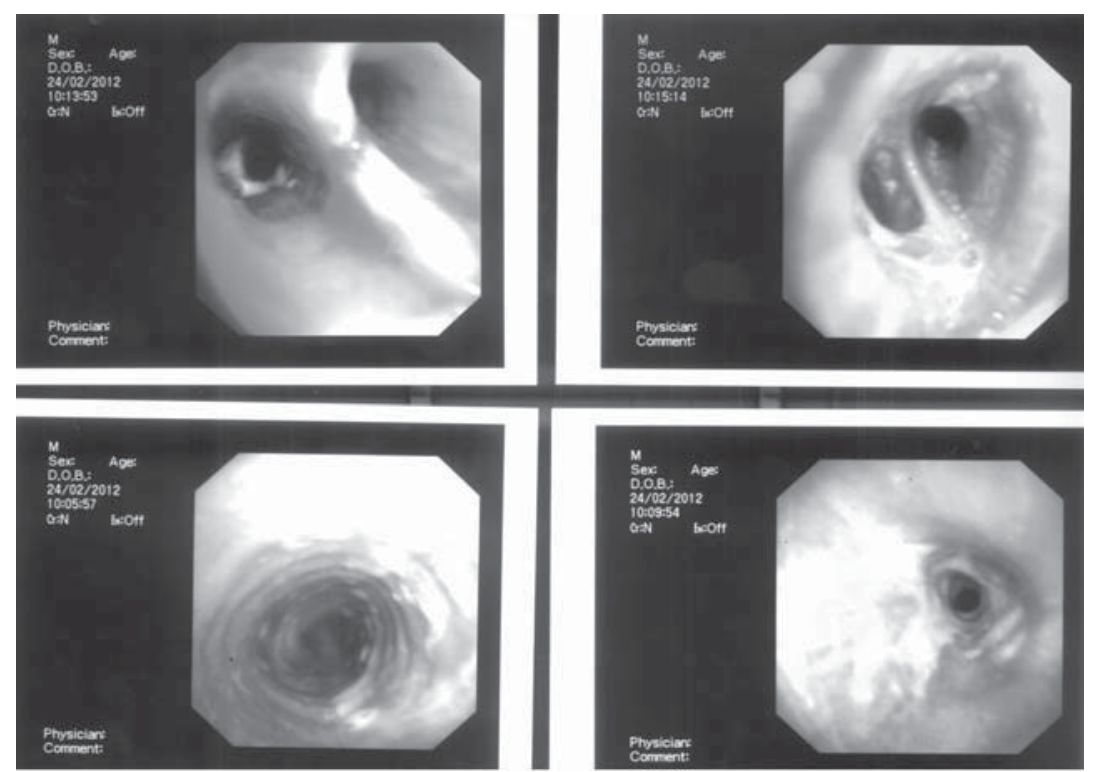

Figure 2. Fiberoptic bronchoscopy showing white traces of barium in the bronchial tree.

of the splenic flexure of the colon with lateroterminal anastomosis, due to a relapse of the stomach GIST in the left subphrenic region. The fistula was diagnosed eight years after the second surgery, with no clinical, radiological and histopatological evidence of a malignant disease, implying that it can be considered as a late post-operative complication.

Thereareafewreportsinliteraturewhich connect surgery and colobronchial fistulas as a complication following coloesophageal interposition, (5) total gastrectomy, (7) or laparoscopic cholecystectomy. (8) In the first two, as in our case, fistulas have been diagnosed years after surgery. What all these cases have in common is the development of the fistula on a damaged site due to previous surgery. In the most recent report, a colobronchial fistula occurred following wedge gastrectomy, splenectomy and distal pancreatectomy, diagnosed twelve months after surgery. (6) Our patient underwent total gastrectomy, splenectomy and distal pancreatectomy due to the stomach GIST three years before the second operation which included splenic flexure resection. We can assume that such extensive operation contributed to the modification of anatomical relations, leaving a wide space for colon loops which facilitated the accretion of the colon to the diaphragm and, under abdominal pressure, a development of the fistula at the site of anastomosis. Colobronchial fistulas are mostly represented with nonspecific respiratory symptoms or recurrent pneumonia. Although in all listed cases fistulas have been developing slowly over a long period, symptoms, when appearing, often may be dramatic with life threatening lung abscesses, sepsis and acute respiratory distress syndrome.

Retrospectively, we found that our patient in the preceding months showed symptoms related to the respiratory system like frequent dry cough and pain in the left lower part of the thorax and left upper region of the abdomen. Patient also displayed an unusually bad breath. These symptoms have been treated as respiratory infections and haven't been connected to previous abdominal surgery. The abdominal MDCT preceding barium enema showed a small infiltrate in the left lower part of the lung and an attracted splenic flexure into the left subphrenic region, without presentation of fistula. Colobronchial fistula has not been recognized until barium enema was made.

Although our patient had mild respiratory symptoms after the barium sulphate had reached the bronchial tree, whichworeoffafterbronchoscopic lavage, cases have been described in which barium aspiration following barium swallow resulted in hypoxemic respiratory failure, with patients dying despite extensive barium suction and resuscitation. $(9,10)$

In conclusion, although colobronchial fistula is a rare condition, it is possible for it to develop years after the surgical resection of the colon in upper abdominal regions. 


\section{REFERENCES}

1. Alameel T, Maclean DA, Macdougall R. Colobronchial fistula presenting with persistent pneumonia in a patient with Crohnžs disease: a case report. Cases J 2009;2:9114.

2. Biswas S, Guy RJ, Phillips RK. Colo-broncho-cutaneous fistula complicating traumatic diaphragmatic rupture. J R Soc Med 2001;94:88-9.

3. Ashley S, Corlett SK, Windle R, Cookson JB. Colobronchial fistula: a late complication of appendicitis. Thorax 1988;43:420-1.

4. Savage PJ, Donovan WN, Kilgore TL. Colobronchial fistula in a patient with carcinoma of the colon. South Med J 1982;75(2):246-7.

5. Zhao X, Sandhu B, Kiev J. Colobronchial fistula as a rare complication of coloesophageal interposition: a unique treatment with a review of the medical literature. Am Surg 2005;71:1058-9.

6. Six CK, Young JS, Sell HW. Image of the month. Colobronchial fistula. Arch Surg 2012;147:573-4.

7. Oyama K, Takagane A, Sone M, Kato K, Kawamura S, Tono C, et al. A case of colobronchial fistula 14 years after total gastrectomy. Jpn J Gastroenterol Surg 2005;38:717-21.

8. Pochin R, Frizzelle F. Colonic-broncho fistula: a previously unreported complication following laparoscopic cholecistectomy. Case Rep Clin Pract Rev 2003;4:77-9.

9. Gernez Y, Barlési F, Doddoli C, Chetaille B, Forel JM, Astoul P, et al. Acute respiratory distress syndrome following inhalation of barium sulfate. Rev Mal Respir 2005;22:477-80.

10. Albeldawi M, Makkar R. Images in clinical medicine. Barium aspiration. N Engl J Med 2012;366:1038. 